\title{
Synthesis and characterization of graphene oxide composite with $\mathrm{Fe}_{3} \mathrm{O}_{4}$
}

\author{
Sun ChUANYU*, WANG Yu \\ Department of Materials Chemistry, Yinuo Institution, Changchun, 130033, China
}

\begin{abstract}
In the paper, a magnetic composite of graphene oxide (MGO) has been successfully synthesized through decomposition of iron (III) acetylacetonate in the mixture solution of triethylene glycol and graphene oxide (GO). Atomic force microscopy (AFM), transmission electron microscopy (TEM), X-ray diffraction (XRD) and superconducting quantum interference device were used to characterize the material. The results show that the magnetic $\mathrm{Fe}_{3} \mathrm{O}_{4}$ nanoparticles modified graphene oxide composite with superparamagnetic properties, and magnetization saturation of $16.4 \mathrm{emu} / \mathrm{g}$ has been obtained. The MGO has a good sustained-release performance, and in vitro cytotoxicity confirming its secure use as a potential drug carrier.
\end{abstract}

Keywords: graphene oxide; composite; magnetic $\mathrm{Fe}_{3} \mathrm{O}_{4}$

(C) Wroclaw University of Technology.

\section{Introduction}

Nanomaterial-based drug carriers have been known as a bridge linking nanotechnology and advanced drug delivery systems because they can allow and realize efficient loading, targeted delivery, and controlled release of drugs [1-4]. Many drugs, especially cancer-therapy drugs, have toxic side effects. Loading these drugs in magnetic carriers could reduce their side effects, because they can make cancer-therapy drugs accumulation in cancer cells at the external magnetic field, thus, reducing the harm to normal cells.

GO has a large specific surface area, and its two hydrophobic polyaromatic basal planes make it possible to adsorb aromatic compounds, making GO an ideal host for drug delivery [4-7]. Recently, graphene oxide modified with iron nanoparticles has attracted much attention due to its promising applications in target drug delivery. Manjunatha et al. [8] firstly coated GO with sodium polystyrene sulfonate, and then obtained magnetic graphene oxide by high temperature decomposition of iron (III) acetylacetonate, and also studied

*E-mail: chuanyuvip@tom.com the applications of magnetic resonance imaging. Chen et al. [9] prepared graphene- $\mathrm{Fe}_{3} \mathrm{O}_{4}$ nanocomposites under hydrothermal conditions, and studied the application of high-performance lithium ion batteries. Yang et al. [10] obtained graphene oxide $-\mathrm{Fe}_{3} \mathrm{O}_{4}$ nanoparticles by chemical precipitation method, loaded doxorubicin hydrochloride on it and studied targeted behavior under an influence of an external magnetic field. Herein, we report a facile method to prepare MGO by in-situ reduction of iron (III) acetylacetonate in the mixture solution of triethylene glycol and GO. The process was very simple, and GO did not need any modification. The toxicity of MGO to HeLa cells was also investigated.

\section{Experimental}

\subsection{Materials}

Native graphite flakes (48 $\mu \mathrm{m}, 99 \%)$ were purchased from Changchun Graphite Limited Company. Iron (III) acetylacetonate $\left(\mathrm{Fe}(\mathrm{acac})_{3}, 99 \%\right)$ was purchased from Sigma. Triethylene glycol (TREG, $99 \%$ ) was obtained from Aldrich. All other reagents used were of analytical grade. 


\subsection{Synthesis of MGO nanoparticles}

Graphene oxide was synthesized from native graphite flakes by a modified Hummers method [11, 12]. Different amounts of the iron precursor $\mathrm{Fe}(\mathrm{acac})_{3}$ were dissolved in $25 \mathrm{~mL}$ TRGE, and then $100 \mathrm{mg}$ of GO was added. After being sonicated for $10 \mathrm{~min}$, the resulting mixture was then heated to $284{ }^{\circ} \mathrm{C}$ at a rate of $10{ }^{\circ} \mathrm{C} \mathrm{min}-1$ under vigorous stirring and argon protection, and the reaction was conducted for $30 \mathrm{~min}$. After cooling to room temperature, $30 \mathrm{~mL}$ ethyl acetate was added to dilute the solution. Then the obtained composite was separated and washed with ethanol several times and dried in vacuum.

\subsection{Characterization}

AFM images were obtained using a Veeco Multimode Pico atomic force microscope in a tapping mode. Transmission electron microscopy (TEM) was performed with a JEOL-2100EX. XRD patterns were obtained using a powder X-ray diffractometer (Rigaku D/MAX-2500/PC). Magnetic properties were investigated by an atomic force microscope (SQUID, MPM-LX).

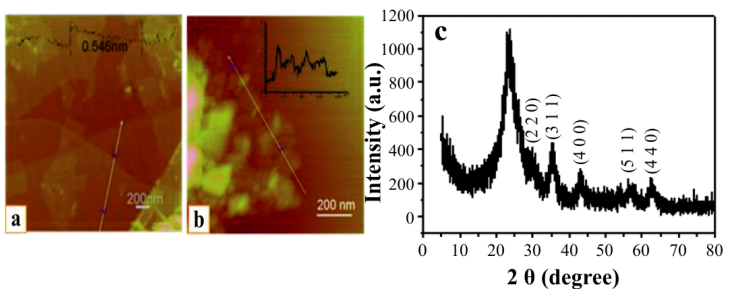

Fig. 1. AFM images of GO (a) and MGO (b), XRD pattern of MGO (c).

\section{Results and discussion}

Fig. 1 shows the AFM images of GO before (a) and after (b) modifying by $\mathrm{Fe}_{3} \mathrm{O}_{4}$. It is found that the obtained graphene oxide has an obvious lamellar structure, with an average height of $0.546 \mathrm{~nm}$. Fig. 1b shows lots of nanoparticles attached on the lamellar structure of GO. Fig. 1c is the XRD pattern of MGO. The diffraction peak at $26^{\circ}$ could be attributed to a native graphite flake. All the other diffraction peaks belong to the cubic inverse spinel
$\mathrm{Fe}_{3} \mathrm{O}_{4}$ - the peaks match well with the (220), (311), (400), (422), (511), and (440) of $\mathrm{Fe}_{3} \mathrm{O}_{4}$.

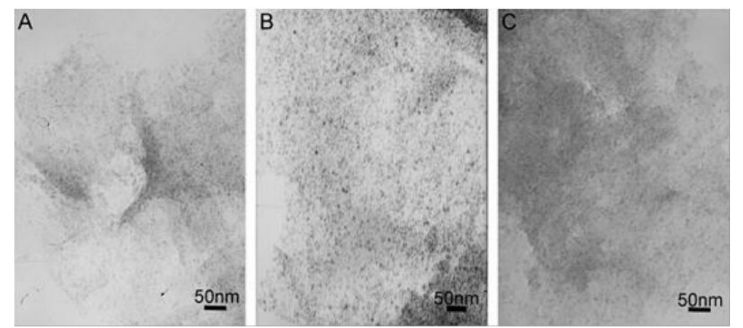

Fig. 2. TEM images of MGO with different quality of iron (III) acetylacetonate: (A) $100 \mathrm{mg}$ (B) 150 $\mathrm{mg}$ (C) $200 \mathrm{mg}$.

The TEM images of MGO are presented in Fig. 2. It is clearly shown that nanoscale $\mathrm{Fe}_{3} \mathrm{O}_{4}$ particles are modified on the GO, and when the quantity of Iron (III) acetylacetonate increases, more $\mathrm{Fe}_{3} \mathrm{O}_{4}$ nanopartilces are attached on GO surface.

After acid oxidation, graphene oxide surface has many oxygen-containing functional groups, such as $\mathrm{OH}, \mathrm{C}=\mathrm{O}, \mathrm{C}-\mathrm{OH}$. The hydrophilic property and static electricity of these groups make GO dispersed in water well. On the other hand, oxygencontaining functional groups are charged with negative electricity, while iron (III) acetylacetonate has a positive charge. So, $\mathrm{Fe}^{3+}$ ions are adsorbed on the surface of GO through electrostatic attraction, and then are restored to $\mathrm{Fe}_{3} \mathrm{O}_{4}$.
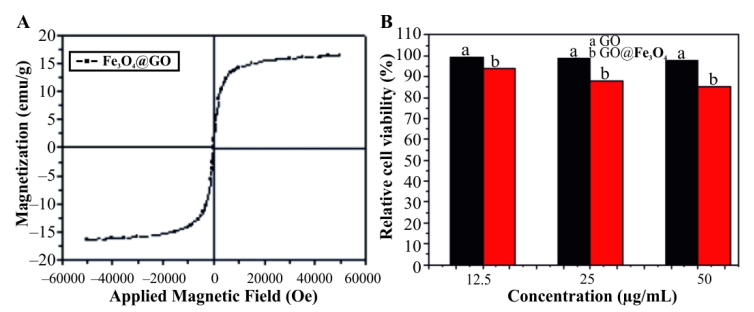

Fig. 3. (A) The room-temperature magnetization curves of MGO, (B) Relative cell viability of GO (a) and MGO (b) at different concentrations.

Magnetic properties of the MGO were investigated with a superconducting quantum interference device (MPM-LX). Fig. 3A shows room temperature magnetization of the MGO. It clearly indicates that the MGO has superparamagnetic properties 
with the saturation magnetization of $16.4 \mathrm{emu} / \mathrm{g}$, which indicates that the material has potential application in magnetic targeting drug delivery systems. MTT assays were performed to evaluate the cytotoxicity of GO and MGO at different concentrations. Fig. 3B shows relative cell viability of GO and MGO at different concentrations. The results reveal that the cytotoxicity of GO can almost be ignored. The cytotoxicity of MGO is slightly higher than that of GO, but as the concentration of MGO increases up to $50 \mu \mathrm{g} / \mathrm{mL}$, cell viability is substantially constant at $88 \%$. Therefore, MGO can be considered as nontoxic.

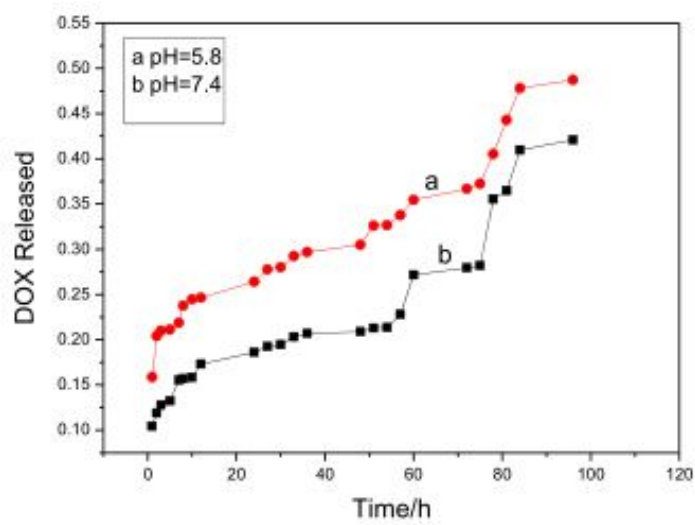

Fig. 4. The release of DOX from MGO at different $\mathrm{pH}$ conditions.

The release behavior of DOX from MGO is shown in Fig. 4. It shows that the MGO has a good sustained-release performance and strong $\mathrm{pH}$ dependence. After $96 \mathrm{~h}$, the cumulative release rate is $48.73 \%$ and $42.11 \%$ at the $\mathrm{pH}$ of 5.8 and 7.4, respectively. This may be due to the stronger hydrogen-bonding interaction between MGO and DOX under basic conditions than that under acid conditions. This effect can be utilized to speed up the drug release inside cancer cells, since the microenvionments in extracellular tissues of tumors and intracellular lysosomes and endosomes are acidic [13].

\section{Conclusions}

In summary, MGO has been successfully prepared through decomposition of iron acetylacetonate in the mixture solution of triethylene glycol and GO. The obtained product showed a superparamagnetic properties with a saturation magnetization value of $16.4 \mathrm{emu} / \mathrm{g}$. In vitro cytotoxicity confirmed that GO and MGO had no cytotoxicity to HeLa cells. The MGO has a great potential application in magnetic targeting drug-delivery technology.

\section{References}

[1] Tian G., Gu Z., LiU X., J. Phys. Chem. C, 115 (48) (2011), 23790

[2] Koo A.N., LeE H.J., Kim S.E., Chem. Commun., 48 (2008), 6570.

[3] Nam T., Park S., LeE S.Y., Bioconjug. Chem., 21 (4) (2010), 578.

[4] Yang X., Zhang X., MA Y., J. Mater. Chem., 19 (18) 92009), 2710.

[5] Zeng L., Li Q., TANG D., Electrochim. Acta, 68 (2012), 158.

[6] Ma X., TaO H., Yang K., Nano Res., 5 (3) (2012), 199.

[7] Hu C., Rong J., Cui J., Carbon, 51 (2013), 255.

[8] Manjunatha R., Suresh G.S., Melo J.S., Sensor. Actuat. B-Chem., 145 (2) (2010), 643.

[9] Chen D., Ji G., MA Y., ACS Appl. Mater. Inter, 3 (8) (2011), 3078.

[10] YAng X.Y., Zhang X.Y., MA Y.F., J. Mater. Chem., 19 (2009), 2710.

[11] Hummers JR. S.W., Offeman R.E., J. Am. Chem. Soc., 80 (1958), 1339.

[12] Li D., Müller M.B., GilJE S., Nat. Nanotechnol., 3 (2) (2008), 101.

[13] Bertorelle F., Wilhelm C., Roger J., Langmuir, 22 (12) 92006), 5385.

Received 2015-05-15

Accepted 2015-04-10 